\title{
Biflatness and biprojectivity of the Fourier algebra
}

\author{
Volker Runde
}

\begin{abstract}
We show that the biflatness - in the sense of A. Ya. Helemskiu- of the Fourier algebra $A(G)$ of a locally compact group $G$ forces $G$ to either have an abelian subgroup of finite index or to be non-amenable without containing $\mathbb{F}_{2}$ as a closed subgroup. An analogous dichotomy is obtained for biprojectivity.
\end{abstract}

Keywords: amenability, biflatness, biprojectivity, Fourier algebra, Leinert set.

2000 Mathematics Subject Classification: Primary 43A30; Secondary 22D99, 43A07, 46J40, 46 M18.

\section{Introduction}

Abstract harmonic analysis is the study of locally compact groups $G$ and the various Banach algebras associated with them, such as the group algebra $L^{1}(G)$. In [9], B. E. Johnson defined the class of amenable Banach algebras to consist of those Banach algebras that satisfy a certain cohomological triviality condition and showed that $L^{1}(G)$ is an amenable Banach algebra if and only if $G$ is an amenable locally compact group in the usual sense ([17]). At about the same time, A. Ya. Helemskil started to systematically develop a homological algebra with functional analytic overtones (see [7] for an account). Amenability in the sense of [9] fits nicely into this framework and is closely related to the notion of biflatness. Another central notion in Helemkiı's theory is that of biprojectivity. Like amenability, the biprojectivity of $L^{1}(G)$ singles out a natural class of groups: $L^{1}(G)$ is biprojective if and only if $G$ is compact ([7, Theorem IV.5.13]).

The Fourier algebra $A(G)$-for arbitrary, not necessarily abelian $G$-was introduced by P. Eymard in [5]. For abelian $G$, we have the dual group $\hat{G}$; in this case, $A(G)$ is nothing but $L^{1}(\hat{G})$ via the Fourier transform. For non-abelian $G$, however, $A(G)$ often displays a behavior strikingly differently from $L^{1}(G)$. For instance, $L^{1}(G \times H) \cong L^{1}(G) \otimes^{\gamma} L^{1}(H)$, with $\otimes^{\gamma}$ denoting the projective tensor product of Banach spaces, holds isometrically isomorphically for all $G$ and $H$ whereas $A(G \times H) \cong A(G) \otimes^{\gamma} A(H)$ holds isomorphically only if $G$ or $H$ is almost abelian, i.e., has an abelian subgroup of finite index ([14]).

Shortly after the publication of [5], H. Leptin provided a characterization of amenability in terms of the Fourier algebra: $G$ is amenable if and only if $A(G)$ has a bounded 
approximate identity ([13]). Since amenable Banach algebras have bounded approximate identities, this suggests that $A(G)$-just like $L^{1}(G)$-is amenable if and only if $G$ is amenable, a view that seemed to have been widespread among mathematicians until the early 1990s. Then, in [10], Johnson showed that there are compact groups $G$, such as $\mathrm{SO}(3)$, for which $A(G)$ fails to be amenable. On the positive side, it is not too difficult to see that $A(G)$ is indeed amenable if $G$ is almost abelian ([11, Theorem 4.1]). Eventually, B. E. Forrest and the author showed that $G$ being almost abelian is not only sufficient but also necessary for $A(G)$ to be amenable ([6, Theorem 2.3]).

Since $A(G)$ is the predual of the von Neumann algebra generated by the left regular representation of $G$, it is an operator space in a canonical manner (see [4] for the theory of operator spaces). As it turns out, $A(G)$ is way better behaved as a completely contractive Banach algebra than as a mere Banach algebra. For instance, $A(G \times H) \cong A(G) \hat{\otimes} A(H)$ holds completely isometrically isomorphically for all $G$ and $H$, where $\hat{\otimes}$ is the projective tensor product of operator spaces, by [4, Theorem 7.2.4]. Johnson's definition of an amenable Banach algebra can be modified to take operator space structures into account, which leads to the notion of operator amenability: this was done in [18], where Z.-J. Ruan showed that $A(G)$ is operator amenable if and only if $G$ is amenable. More generally, Helemskiul's Banach homology can be developed in an operator space context as well (see [1], for instance), which leads to further interesting results about $A(G)$ : it is operator biprojective if and only if $G$ is discrete ([1], [19], or [21]), and it is operator biflat for all so-called [SIN]-groups ([19]) and possibly for all $G([2])$.

In this brief note, we take a look at $A(G)$ in the framework of Helemskiü's original Banach homology. We are interested in the properties of $G$ that are implied by the biflatness and biprojectivity of $A(G)$, respectively. Of course, $A(G)$ is biflat if $G$ is almost abelian. Our main result is that if $A(G)$ is biflat and $G$ is not almost abelian, then $G$ is non-amenable, but does not contain $\mathbb{F}_{2}$, the free group in two generators, as a closed subgroup.

\section{The result}

We begin with recalling the definitions of biprojectivity, biflatness, and amenability.

Given a Banach algebra $\mathfrak{A}$, we let $m: \mathfrak{A} \otimes^{\gamma} \mathfrak{A} \rightarrow \mathfrak{A}$ denote the multiplication map, i.e., $m(a \otimes b)=a b$ for $a, b \in \mathfrak{A}$; if we want to emphasize the algebra, we sometimes write $m_{\mathfrak{A}}$. The tensor product $\mathfrak{A} \otimes^{\gamma} \mathfrak{A}$ becomes a Banach $\mathfrak{A}$-bimodule via

$$
a \cdot(x \otimes y):=a x \otimes y \quad \text { and } \quad(x \otimes y) \cdot a:=x \otimes y a \quad(a, x, y \in \mathfrak{A})
$$

turning $m$ into a bimodule homomorphism.

Definition 1. Let $\mathfrak{A}$ be a Banach algebra. Then $\mathfrak{A}$ is called 
(a) biprojective if $m: \mathfrak{A} \otimes^{\gamma} \mathfrak{A} \rightarrow \mathfrak{A}$ has a bounded bimodule right inverse,

(b) biflat if $m^{*}: \mathfrak{A}^{*} \rightarrow(\mathfrak{A} \otimes \gamma \mathfrak{A})^{*}$ has a bounded bimodule left inverse, and

(c) amenable if it is biflat and has a bounded approximate identity.

Remark. These definitions are not the original ones due to Johnson and Helemskiı̌, respectively, but are equivalent to them ([7]).

In order to deduce consequences for the structure of $G$ from the biflatness or biprojectivity of $G$, respectively, we require the following definition due to M. Leinert ([12]):

Definition 2. Let $G$ be a discrete group. A subset $E$ of $G$ is called a Leinert set if $\chi_{E} A(G) \cong \ell^{2}(E)$ holds isomorphically.

Here, $\chi_{E}$ denotes the indicator function of $E$.

Trivially, all finite sets are Leinert sets. Remarkably, however, $\mathbb{F}_{2}$ contains infinite Leinert set: this is the main result of [12]. As was observed in [12], this implies that $A\left(\mathbb{F}_{2}\right)$ does not factor, i.e., there are functions in $A\left(\mathbb{F}_{2}\right)$ that are not product of two functions in $A\left(\mathbb{F}_{2}\right)$. More is true:

Lemma. Let $G$ be a locally compact group containing $\mathbb{F}_{2}$ as a closed subgroup. Then $m: A(G) \otimes^{\gamma} A(G) \rightarrow A(G)$ is not surjective.

Proof. Assume that $m_{A(G)}$ is surjective.

For any closed $F \subset G$, let $\rho_{F}$ denote the restriction map from $A(G)$, i.e., a function in $A(G)$ is restricted to $F$. By $\left[8\right.$, Theorem 16], $\rho_{\mathbb{F}_{2}}$ maps $A(G)$ onto $A\left(\mathbb{F}_{2}\right)$. Since the diagram

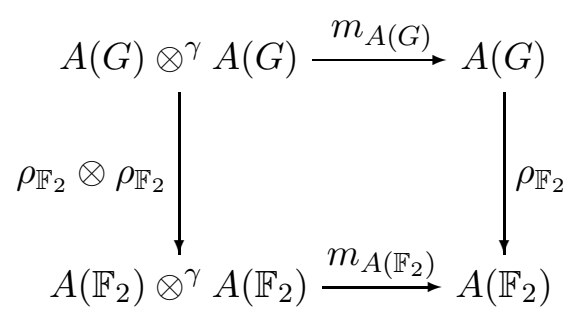

commutes, and since its columns are surjective, we conclude that $m_{A\left(\mathbb{F}_{2}\right)}$ is surjective as well.

Let $E \subset \mathbb{F}_{2}$ be an infinite Leinert set. By Definition 2, is is clear that $\rho_{E}$ maps $A\left(\mathbb{F}_{2}\right)$ onto $\ell^{2}(E)$. Again, we have a commutative diagram

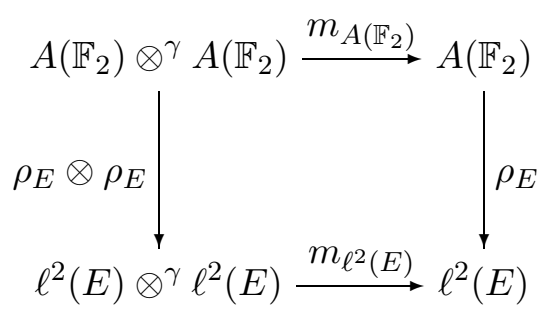


with surjective columns, so that $m_{\ell^{2}(E)}$ has to be surjective, too. Since the range of $m_{\ell^{2}(E)}$ is contained in $\ell^{1}(E) \subsetneq \ell^{2}(E)$ by the Cauchy-Schwarz inequality, this yields a contradiction.

Remark. For $G=\mathbb{F}_{2}$, the Lemma was already obtained, but never published, by $\mathrm{H}$. Steiniger ([20]) with a somewhat more technical proof that does not explicitly use Leinert sets.

Proving our main result now requires little more than assembling the right bits and pieces:

Theorem. Let $G$ be a locally compact group such that $A(G)$ is biflat. Then one of the following holds:

(a) $G$ is almost abelian.

(b) $G$ does not contain $\mathbb{F}_{2}$ as a closed subgroup, but fails to be amenable.

Proof. Suppose that $G$ is amenable. Then $A(G)$ has a bounded approximate identity by [13], making $A(G)$ amenable. By [6, Theorem 2.3], this means that (a) holds.

Suppose that $G$ is not amenable, and assume towards a contradiction that $G$ contains $\mathbb{F}_{2}$ as a closed subgroup. By the definition of biflatness, $m^{*}: A(G)^{*} \rightarrow\left(A(G) \otimes^{\gamma} A(G)\right)^{*}$ has a bounded left inverse and thus, in particular, is injective with closed range. Consequently, $m: A(G) \otimes^{\gamma} A(G) \rightarrow A(G)$ has to be surjective, which contradicts the Lemma. Hence, (b) must hold.

Remark. The question of whether or not (discrete) groups as in (b) exist was open for several decades, and the belief that no such groups exist became known as "von Neumann's conjecture". Eventually, A. Yu. Ol'shanskil came up with a counterexample to this conjecture ([16]), so that condition (b) is not vacuous.

As biprojectivity is stronger than biflatness, the conclusions of the Theorem apply, in particular, if $A(G)$ is biprojective. Furtheremore, by general Banach algebra theory ( $\underline{3}$, Corollary 2.8.42]), the biprojectivity of $A(G)$ forces $G$ to be discrete.

We summarize:

Corollary. Let $G$ be a locally compact group group such that $A(G)$ is biprojective. Then $G$ is discrete, and one of the following holds:

(a) G is almost abelian.

(b) $G$ does not contain $\mathbb{F}_{2}$ as a closed subgroup, but fails to be amenable.

Furthermore, for any discrete $G$, (a) is also sufficient for $A(G)$ to be biprojective. 
Proof. Only the "furthermore" part still needs consideration. Suppose that $G$ is discrete and almost abelian. Then $A(G)$ is amenable, and since $A(G)$ is Tauberian, the discreteness of $G$ forces multiplication in $A(G)$ to be compact. By [15, Corollary 3.2], this means that $A(G)$ is biprojective.

\section{References}

[1] O. Yu. Aristov, Biprojective algebras and operator spaces. J. Math. Sci. (New York) 111 (2002), 3339-3386.

[2] O. Yu. Aristov, V. Runde, and N. Spronk, Operator biflatness of the Fourier algebra and approximate indicators for subgroups. J. Funct. Anal. 209 (2004), 367-387.

[3] H. G. Dales, Banach Algebras and Automatic Continuity. London Mathematical Society Monographs (New Series) 24, Clarendon Press, Oxford, 2000.

[4] E. G. Effros and Z.-J. Ruan, Operator Spaces. London Mathematical Society Monographs (New Series) 23, Clarendon Press, Oxford, 2000.

[5] P. Eymard, L'algèbre de Fourier d'un groupe localement compact. Bull. Soc. Math. France 92 (1964), 181-236.

[6] B. E. Forrest and V. Runde, Amenability and weak amenability of the Fourier algebra. Math. Z. 250 (2005), 731-744.

[7] A. Ya. HelemskiI, The Homology of Banach and Topological Algebras (translated from the Russian). Mathematics and Its Applications (Soviet Series) 41, Kluwer Academic Publishers Group, Dordrecht, 1989.

[8] C. Herz, Harmonic synthesis for subgroups. Ann. Inst. Fourier (Grenoble) 23 (1973), 91123.

[9] B. E. Johnson, Cohomology in Banach algebras. Mem. Amer. Math. Soc. 127 (1972).

[10] B. E. Johnson, Non-amenability of the Fourier algebra of a compact group. J. London Math. Soc. (2) 50 (1994), 361-374

[11] A. T.-M. LaU, R. J. Loy, and G. A. Willis, Amenability of Banach and $C^{*}$-algebras on locally compact groups. Studia Math. 119 (1996), 161-178.

[12] M. Leinert, Faltungsoperatoren auf gewissen diskreten Gruppen. Studia Math. 52 (1974), $149-158$.

[13] H. Leptin, Sur l'algèbre de Fourier d'un groupe localement compact. C. R. Acad. Sci. Paris, Sér. A 266 (1968), 1180-1182.

[14] V. Losert, On tensor products of Fourier algebras. Arch. Math. (Basel) 43 (1984), 370-372.

[15] R. J. Loy, C. J. Read, V. Runde, and G. A. Willis, Amenable and weakly amenable Banach algebras with compact multiplication. J. Funct. Anal. 171 (2000), 78-114.

[16] A. Yu. Ol'shanskiI , On the problem of the existence of an invariant mean on a group (in Russian). Russian Math. Surveys 35 (1980), 180-181. 
[17] A. L. T. Paterson, Amenability. Mathematical Surveys and Monographs 29, American Mathematical Society, Providence, RI, 1988.

[18] Z.-J. RuAn, The operator amenability of $A(G)$. Amer. J. Math. 117 (1995), 1449-1474.

[19] Z.-J. RUAN and G. XU, Splitting properties of operator bimodules and operator amenability of Kac algebras. In: A. Gheondea, R. N. Gologan, and D. Timotin (ed.s), Operator Theory, Operator Algebras and Related Topics, pp. 193-216. The Theta Foundation, Bucharest, 1997.

[20] H. Steiniger, Finite-dimensional extensions of Fourier algebras. Preprint, University of Leeds, 1997.

[21] P. J. Wood, The operator biprojectivity of the Fourier algebra. Canad. J. Math. 54 (2002), $1100-1120$.

[October 26, 2018]

Author's address: Department of Mathematical and Statistical Sciences

University of Alberta

Edmonton, Alberta

Canada T6G 2G1

E-mail: $\quad$ vrunde@ualberta.ca

URL: $\quad$ http://www.math.ualberta.ca/ runde/ 